Check for updates

Cite this: Chem. Sci., 2019, 10, 1677

๑ All publication charges for this article have been paid for by the Royal Society of Chemistry

Received 28th September 2018 Accepted 24th November 2018

DOI: $10.1039 / \mathrm{c} 8 \mathrm{sc} 04318 \mathrm{c}$

rsc.li/chemical-science

\section{The biobehavior, biocompatibility and theranostic application of SPNS and PdaAu nanoplates in rats and rabbits $\uparrow$}

\author{
Jingchao Li, ${ }^{a}$ Hongzhi Liu, ${ }^{\mathrm{c}}$ Jiang Ming, ${ }^{a}$ Duo Sun, ${ }^{a}$ Xiaolan Chen, (D) *ab \\ Xiaolong Liu (D) ${ }^{c}$ and Nanfeng Zheng (iD) *a
}

On account of the fascinating surface plasmon resonance (SPR) properties, the ability of passively targeting tumors and remarkable biocompatibility, two-dimensional (2D) Pd-based nanomaterials have demonstrated wide application prospects in cancer theranostics. However, the used animal models for exploring the bioapplications and biosafety of 2D Pd-based nanomaterials were usually limited to mice. To further widen their biomedical applications and promote future clinical transformation, it is necessary to make a breakthrough in animal models. In this work, Sprague Dawley (SD) rats and New Zealand rabbits were used as the experimental animals and orthotopic liver tumors or subcutaneous tumors were induced in these animals. Taking $\approx 5 \mathrm{~nm}$ small Pd nanosheets (SPNS) and $30 \mathrm{~nm}$ Pd@Au nanoplates $(\mathrm{Pd}(\mathrm{Au})$ as the representative 2D Pd-based nanomaterials, we investigated their biobehaviors and biosafety in rat liver $\&$ subcutaneous tumor models and rabbit liver tumors. The results indicated that SPNS and PdaAu could still effectively accumulate on the tumor sites of these bigger animal models by the enhanced permeability and retention (EPR) effect, and the accumulation effects were closely related to their sizes. Metabolism studies confirmed that SPNS could be excreted out of rats through urine. Moreover, based on the sufficient uptake by cancer cells and passive accumulation of SPNS and Pd@Au in subcutaneous tumors in rats, we performed photothermal therapy (PTT) in vitro and in vivo. Significant tumor growth inhibition illustrated that even though the animal model was dozens of times bigger than the mouse model, the 2D Pd-based nanomaterials satisfied the requirements of being an outstanding photothermal reagent. Finally, the hematological and histological examination results suggested that SPNS and Pd@Au had favorable biocompatibility in rats and rabbits at a given dose. We hope this work will drive the development of 2D Pd-based nanomaterials towards practical clinical applications and provide a guide for other theranostic nanoplatforms that will be applied in bigger animal tumor models in the future.
${ }^{a}$ State Key Laboratory for Physical Chemistry of Solid Surfaces, Collaborative Innovation Center of Chemistry for Energy Materials, National \& Local Joint Engineering Research Center of Preparation Technology of Nanomaterials, College of Chemistry and Chemical Engineering, Xiamen University, Xiamen 361005, China. E-mail: chenxl@xmu.edu.cn; nfzheng@xmu.edu.cn

${ }^{b}$ State Key Laboratory for Chemistry and Molecular Engineering of Medicinal Resources, Guangxi Normal University, Guilin 541004, China

'The United Innovation of Mengchao Hepatobiliary Technology Key Laboratory of Fujian Province, Mengchao Hepatobiliary Hospital of Fujian Medical University, Fuzhou, 350025, China

$\dagger$ Electronic supplementary information (ESI) available: The photos of the rabbit orthotopic liver tumor, and orthotopic liver tumor and subcutaneous tumor of rats, molar absorption coefficients of SPNS at different wavelengths, TEM images of Pd@Au-PEG and Pd@Au-PEG after laser irradiation, the biodistribution results of SPNS and Pd@Au in rats and rabbits calculated in \% ID, accumulation amount changes of SPNS in rat kidney, excretion of Pd@Au by feces, loading efficiencies of Cy5.5 on SPNS and Pd@Au, color changes of rat subcutaneous tumors after intravenous injection of SPNS and Pd@Au, cellular uptake of SPNS and Pd@Au by W256 cells and VX2 cells, and PTT efficiency for in vitro killing VX2 cells. See DOI: 10.1039/c8sc04318c

\section{Introduction}

With the advantage of size which is close to those of some biological molecules like DNA, enzymes and proteins, nanomaterials have been widely used in the biomedical field. ${ }^{1}$ Therein, the application of nanomaterials in cancer theranostics has become a hot research focus and attracted much attention. $^{2-5}$ Many nanomaterial-based theranostic platforms have been reported to greatly improve the imaging and therapeutic efficiency for cancer. ${ }^{6-12}$ To evaluate the theranostic effects as well as biosafety of the designed nanoplatforms, certain animal models need to be adopted. In general, mice are common and widely used laboratory animals because they are mammals like humans, and the price of mice is low and they can reproduce quickly. ${ }^{13}$ The strains of mice for experimental use contain BALB/C, C57BL/6, DBA/2 and so on. ${ }^{14-17}$ Different types of tumors including subcutaneous tumors, orthotopic liver tumors and metastatic tumors could be induced on these 
experimental mice by xenograft or direct induction. ${ }^{\mathbf{1 8 , 1 9}}$ Many nanomaterials demonstrated wonderful enrichment performance in these tumor models by the enhanced permeability and retention (EPR) effect and had excellent biosafety in mice. ${ }^{\mathbf{2 0}, 21}$ However, humans and mice still have a huge body size gap. Whether the EPR effect can still be well reflected and the biosafety of nanomaterials remains in bigger animal models are worth further study and exploration.

Generally speaking, the biobehaviors of rats and rabbits are closer to those of humans than mice, so researchers usually take rats or rabbits as objects to study the pharmacokinetics and toxicology of a number of nanodrugs in the field of translational medicine. ${ }^{22-24}$ However, using rats or rabbits as amplifying animal models to investigate the theranostic effects of some functional nanomaterials is rarely reported. In 2014, Terentyuk et al. utilized Au nanorods with a hematoporphyrin-loaded silica shell for dual-modality photothermal and photodynamic treatments (PTT + PDT) of rat subcutaneous tumors in vivo. ${ }^{25}$ More recently, Tan et al. reported layered $\mathrm{MoS}_{2}$ hollow spheres for PTT of rabbit liver orthotopic tumors. ${ }^{26}$ Their results fully demonstrated that the tumor growths could be effectively suppressed by single or combined therapy in these bigger animal tumor models. Nevertheless, more detailed in vivo biobehavior explorations, such as blood circulation and tissue distribution of these nanomaterials in rats or rabbits, were not given in these studies.

Recently, 2D Pd-based nanomaterials have been reported to be highly efficient PTT agents, carriers of chemotherapeutic drugs and photosensitizers, and retain admirable biocompatibility in mice. ${ }^{27-29}$ Among them, small Pd nanosheets (SPNS) with an ultra-small size $(\approx 5 \mathrm{~nm})$ can effectively accumulate in the tumor sites of mice and be eliminated by renal clearance. ${ }^{30}$ And 30 nm Pd@Au nanoplates also can target mouse tumors

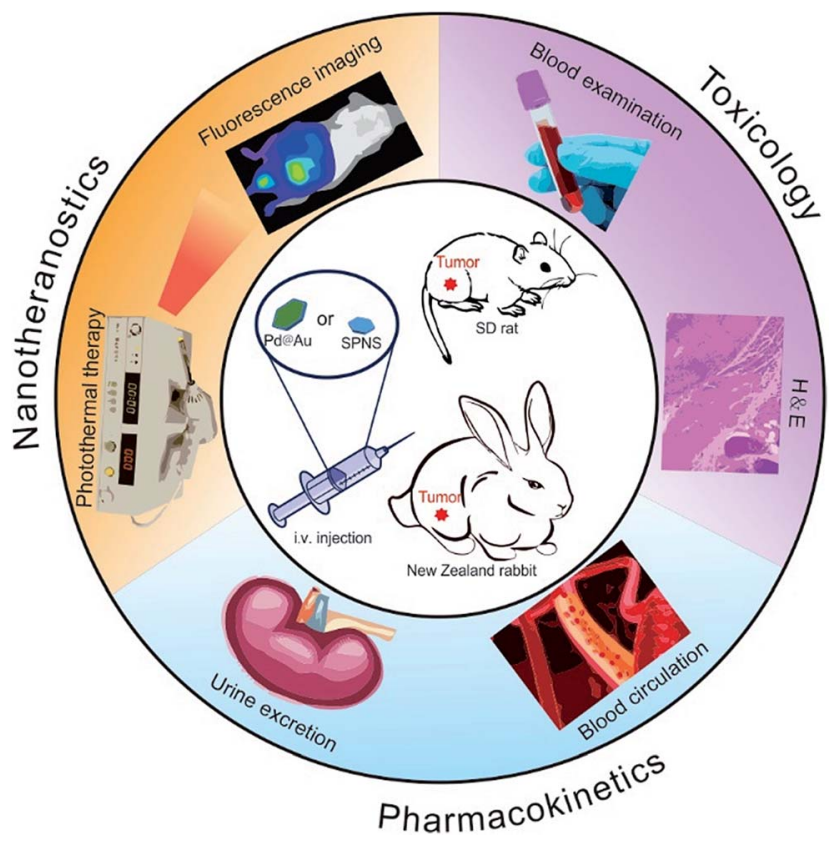

Scheme 1 The overview of in vivo biobehaviors, theranostic effects and biosafety of SPNS and Pd@Au in rats and rabbits. with high efficiency by the EPR effect, and the accumulation amount in tumors reached $79 \%$ ID (injection dose) per gram. ${ }^{31}$ Herein, by successfully constructing the rat and rabbit tumor models (Fig. S1 $\dagger$ ), we innovatively and systematically investigated the living biobehaviors, theranostic effects and biosafety of SPNS and Pd@Au in these bigger animal models (Scheme 1). Surprisingly, both SPNS and $30 \mathrm{~nm}$ Pd@Au could precisely identify tumor lesions either in the liver or subcutaneous sites of rats or rabbits measured by Inductively Coupled Plasma Mass Spectrometry (ICP-MS), and fluorescence and photoacoustic (PA) imaging. And the sizes of SPNS and Pd@Au also had an important impact on their blood circulation half-lives and urinary metabolic efficiency in rats. With the good enrichment of SPNS and Pd@Au in tumors, the complete elimination of subcutaneous tumors in rats can be achieved through PTT. Meanwhile blood biochemical analyses and the histological section results indicated the favorable biosafety of SPNS and $30 \mathrm{~nm}$ Pd@Au in rats and rabbits. Our work showed that $2 \mathrm{D}$ Pd-based nanomaterials have great potential for future clinical applications.

\section{Results and discussion}

\section{Synthesis and characterization of SPNS and Pd@Au}

Small Pd nanosheets (SPNS, $\approx 5 \mathrm{~nm}$ ) and Pd@Au nanoplates (Pd@Au, $\approx 30 \mathrm{~nm}$ ) were prepared according to our previously published procedures with slight modification. ${ }^{30,31}$ As shown in the Transmission electron microscopy (TEM) images of Fig. 1a and $b$, the as-prepared SPNS and Pd@Au are hexagonal with uniform sizes. From Fig. 1c and d, both SPNS and $30 \mathrm{~nm} \mathrm{Pd@Au}$ exhibited strong absorbance in the near infrared (NIR) region.
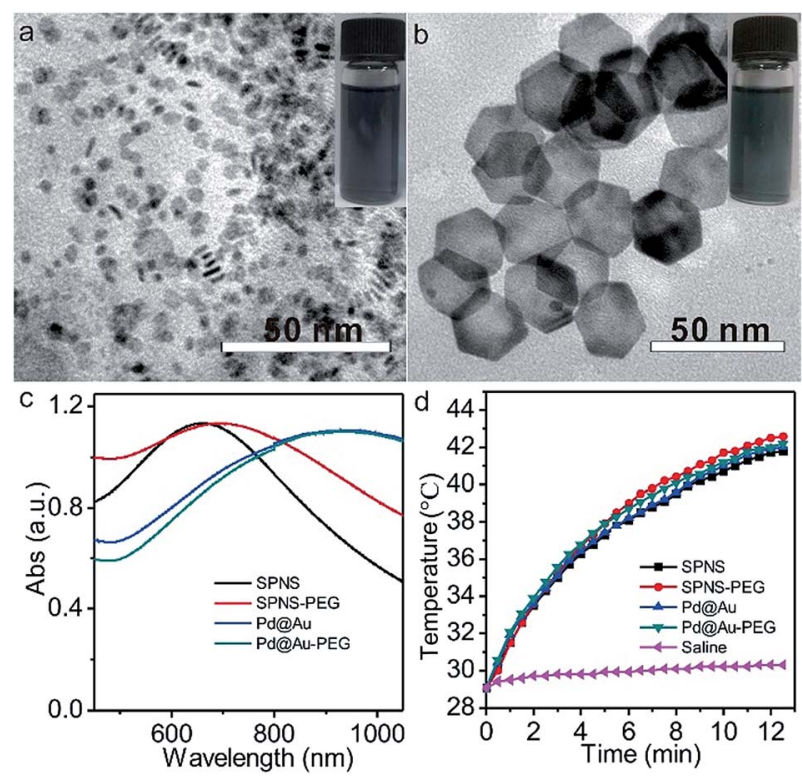

Fig. 1 The characterization of nanomaterials: TEM images of SPNS (a) and Pd@Au (b), and UV-Vis-NIR absorption spectra (c) and photothermal rising temperature effects (d) of SPNS, SPNS-PEG, Pd@Au and Pd@Au-PEG (concentrations of nanomaterials: $50 \mathrm{ppm}$ ). The insets in ( $a$ and $b$ ) show the photos of SPNS and Pd@Au aqueous solutions, respectively, and the laser power density is $0.5 \mathrm{~W} \mathrm{~cm}^{-2}(808 \mathrm{~nm})$. 
The corresponding molar absorption coefficients of SPNS (40 $\mathrm{ppm}$ ) at different wavelengths were also measured based on the Beer-Lambert Law and the $\varepsilon$ value could reach around $1534 \mathrm{~L}$ $\mathrm{mol}^{-1} \mathrm{~cm}^{-1}$ at a wavelength of $808 \mathrm{~nm}$ (Fig. S2 $\dagger$ ). To extend the blood circulation half-lives of SPNS and Pd@Au in rats and rabbits and compare the in vivo results with those in mice models, SPNS and Pd@Au were further surface-modified with thiol-polyethylene glycol (mPEG-SH) to obtain SPNS-PEG and Pd@Au-PEG, respectively. The morphologies, sizes and absorption spectra of SPNS-PEG and Pd@Au-PEG were similar to those of the corresponding SPNS and Pd@Au (Fig. 1c and $\mathrm{S} 3 \uparrow$ ). And after laser irradiation for $10 \mathrm{~min}$, the morphology of Pd@Au-PEG was still maintained well which demonstrated their good photothermal stability (Fig. S4†). Therefore, the excellent absorptions of SPNS-PEG and Pd@Au-PEG in the NIR region together with their high photothermal conversion capability (Fig. 1d), stability and good biocompatibility endowed them with great potential for application in cancer theranostics.

\section{In vivo pharmacokinetics, excretion and biodistribution studies in rats bearing an orthotopic liver tumor model}

As demonstrated above, 2D Pd-based nanomaterials with high photothermal conversion efficiency and good biocompatibility have brilliant prospects for application in cancer PTT and PTTbased combined therapy. However so far, the in vivo behavior explorations and theranostic applications of 2D Pd-based nanomaterials are still limited to mice. To further promote the biomedical applications of Pd-based 2D nanomaterials, more animal models are required to assess their biosafety and theranostic effects. Here, we first investigated the blood circulation, biodistribution and urinary excretion of SPNS and Pd@Au in rats. In our previous work, it was found that the blood circulations of 2D Pd-based nanomaterials in mice were closely related to their sizes and surface modifications. ${ }^{28,32}$ A similar size-dependent circulation time was also observed in rats. As shown in Fig. 2a, the circulation half-lives of SPNS-PEG and Pd@Au-PEG in rats by measuring the Pd concentrations in blood by ICP-MS were $10 \mathrm{~h}$ and $2.5 \mathrm{~h}$, respectively. It was easier for SPNS with a super small size $(\approx 5 \mathrm{~nm})$ to escape the capture of the reticuloendothelial system (RES), so the blood circulation time of SPNS was longer than that of $30 \mathrm{~nm}$ Pd@Au. The long circulation half-life also gave nanoparticles more contact time to accumulate in the target tissues. Fig. $2 \mathrm{c}$ and $\mathrm{d}$ show the biodistribution results of SPNS and Pd@Au in rats with the orthotopic liver tumor model which was fabricated by surgical operation and injection of cancer cells, respectively. Owing to their ultra-small size as well as long circulation time, SPNS had a relatively broad distribution in various organs, including the heart, liver, spleen, lung, kidney and tumor. Especially the high concentration in kidneys indicated that SPNS might be excreted out of the body through the urinary system. Compared to SPNS, the larger-sized Pd@Au mainly accumulated in the spleen. It was worth noting that the accumulations of SPNS and Pd@Au in the orthotopic liver tumors increased with time. At $48 \mathrm{~h}$ post injection, the accumulation amounts were $2.03 \%$ ID per $g$ and
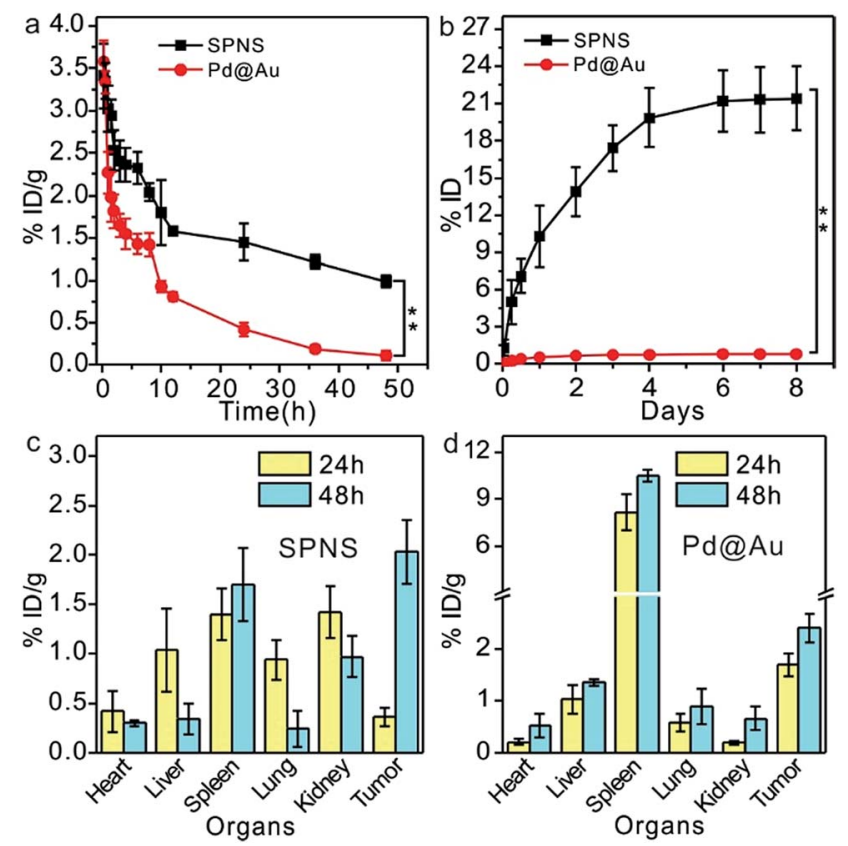

Fig. 2 The investigation of biobehavior in vivo ( $n=4$ for each group): blood circulation (a) and urinary excretion (b) of SPNS-PEG and Pd@Au-PEG; biodistribution of SPNS-PEG (c) and Pd@Au-PEG (d) in rats with the orthotopic liver tumor model. Asterisks suggest statistically significant differences $(* P<0.05, * * P<0.01$, and $* * * P<0.001)$.

2.41\% ID per $\mathrm{g}$ for SPNS and Pd@Au, respectively (Fig. 2c and d). Moreover, the biodistribution results that used \% ID as the unit are also presented in Fig. S5(a and $b \dagger$ ). The size effect was also apparent in urinary excretion. It is generally recognized that nanomaterials that are less than $10 \mathrm{~nm}$ in size would be excreted out from the glomerulus through urine. ${ }^{33,34}$ In our study, 10.3\% ID SPNS was excreted out from the body through urine within one day but some $(0.56 \%$ ID) Pd@Au could be detected in urine during the same period and the amount remained invariable (Fig. 2b). For SPNS, the metabolic rate started to go down after 2 days and the total amount of SPNS excreted out through urine within 8 days was about $22 \%$ ID. The trend was also confirmed by the accumulation amount changes of SPNS in the kidney with time (Fig. S6†). Furthermore, the possible metabolic pathway of Pd@Au was also further investigated, and about 9\% ID of Pd@Au could be excreted out from the body through feces within 8 days (Fig. S7†), indicating the larger-sized Pd@Au could not be effectively cleared out of the body through urine and feces metabolism routes. The feature of SPNS that can be easily eliminated out of the body via urine enables it to be an ideal nanoplatform for application in cancer theranostics.

\section{In vivo biodistribution of SPNS and Pd@Au in rat subcutaneous tumor model}

Due to the simplicity of fabrication, the subcutaneous tumor model was frequently used in mice to appraise the performance of the theranostic nanoplatform. For better comparison with the results in the mouse subcutaneous tumor model, we further 
investigated the biodistribution of SPNS and Pd@Au in the rat subcutaneous tumor model. Fluorescence imaging is widely used for tumor diagnosis because of its high resolution and sensitivity. With facile surface functionalization, 2D Pd-based nanosheets (NSs) could serve as a suitable fluorescence probe carrier for in vitro and vivo fluorescence imaging. By way of covalent linkage, Cy5.5 modified NSs were successfully synthesized and intravenously injected into rats. The loading efficiencies of Cy5.5 on SPNS (18.8\%,w/w) and Pd@Au (25.6\%, w/ w) were calculated by the difference of adding Cy5.5 and the Cy5.5 in the supernatant after purification according to the standard absorption curve of Cy5.5 at $678 \mathrm{~nm}$ (Fig. S8†). As shown in Fig. 3a, free dye could not accurately target subcutaneous tumor sites so the fluorescence signal tended to be dispersive in the scanned area. Obviously, the intensity of the signal at tumor sites was not higher than that in the normal area. However, SPNS-Cy5.5 or Pd@Au-Cy5.5 by tail vein injection rapidly accumulated in tumors and there was a strong fluorescence signal at $24 \mathrm{~h}$ post injection. As time went by, signals at tumor sites became stronger at $48 \mathrm{~h}$ than before. The fluorescence imaging results not only indicated that SPNS and Pd@Au had the potential to be the carriers of some molecule imaging contrast agents, but also qualitatively confirmed that the SPNS and Pd@Au possessed nice tumor passive targeting ability in rats. Moreover, after intravenous injection of nanomaterials, the subcutaneous tumor sites obviously grew black

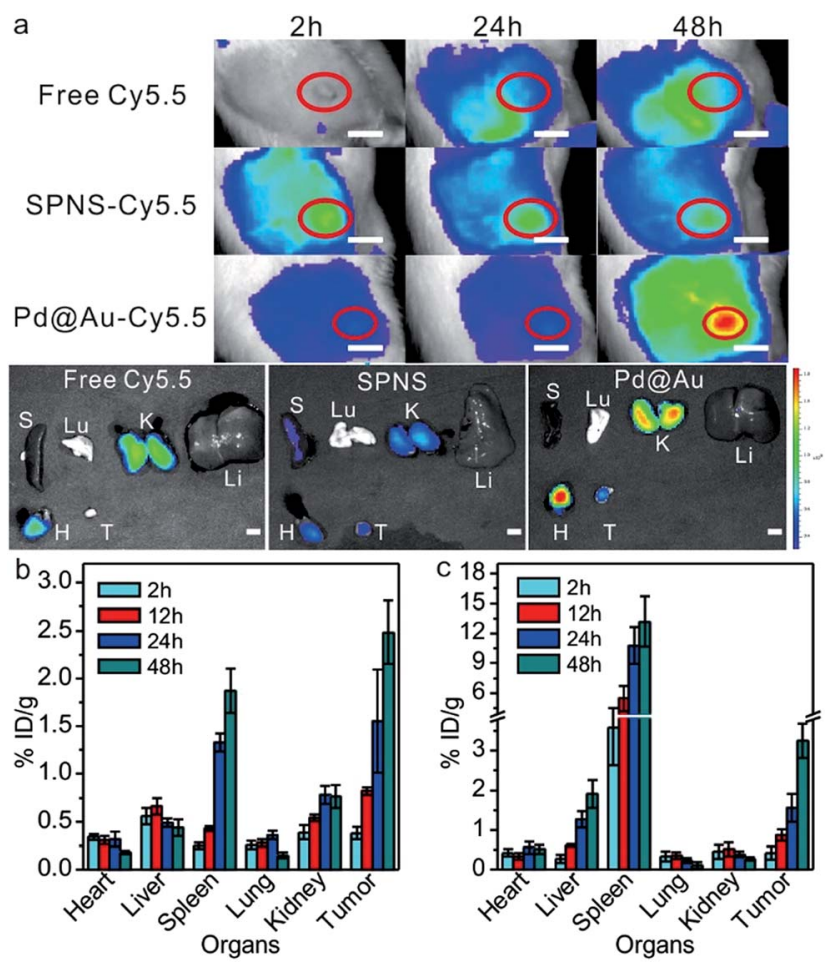

Fig. 3 Live fluorescence imaging and signals in major tissues $(\mathrm{H}$ : heart, Li: liver, S: spleen, Lu: lung, K: kidney and T: tumor) of Cy5.5, SPNSCy5.5 and Pd@Au-Cy5.5 (a), respectively. The red circles represent the location of the tumors. Biodistribution of SPNS-PEG (b) and PdaAu$P E G(c)$ in the rat subcutaneous tumor model ( $n=4$ for each group). In all fluorescence images, the amounts of Cy5.5 in different groups were $200 \mu \mathrm{g}$. The scale bar in a is $1 \mathrm{~cm}$. within 6 h for SPNS (Fig. S9†) and 24 h for Pd@Au (Fig. S10†), which more intuitively showed the specific accumulation of SPNS and Pd@Au in target tissue.

Meanwhile, the precise quantitation of biodistribution in the rat subcutaneous tumor model by ICP-MS was also performed. As seen from Fig. 3b and c, the accumulation amounts of SPNS or $30 \mathrm{~nm}$ Pd@Au in tumors kept continuously increasing at $2 \mathrm{~h}$, $12 \mathrm{~h}, 24 \mathrm{~h}$ and $48 \mathrm{~h}$ post injection, which was similar to that in the orthotopic liver tumor model due to the similar internal structures of these two tumor models. And at $48 \mathrm{~h}$ the enrichment reached $2.48 \%$ ID per $g$ for SPNS and $3.25 \%$ ID per $g$ for Pd@Au, respectively (the \% ID distribution data are also shown in Fig. S5(c and $d \dagger)$ ). These results further suggested that both SPNS and $30 \mathrm{~nm}$ Pd@Au had fantastic passive targeting ability to recognize lesions in the subcutaneous tumor of rats.

\section{In vitro and in vivo PTT of tumor cells and subcutaneous tumors of rats}

The excellent absorptions in the NIR region of SPNS and Pd@Au together with their abilities of passively targeting tumors could
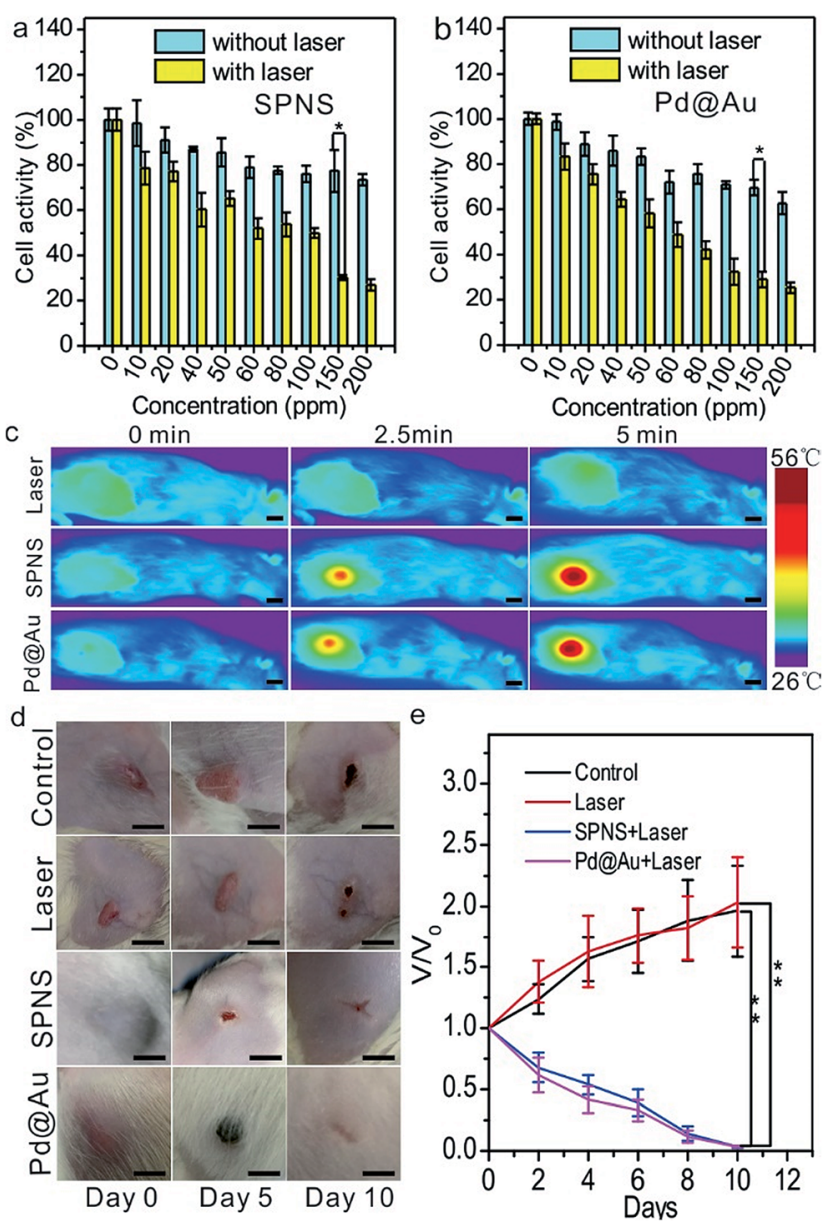

Fig. 4 In vitro PTT of SPNS (a) and Pd(aAu (b) ( $n=8$ for each group). The temperature increases of tumor sites upon laser irradiation after intravenous injection with SPNS and Pd@Au (c). In vivo PTT of SPNS and Pd@Au (d) and the tumor growth curves in different treatment groups (e) ( $n=4$ for each group). Asterisks suggest statistically significant differences $(* P<0.05, * * P<0.01$, and $* * * P<0.001)$. 
a

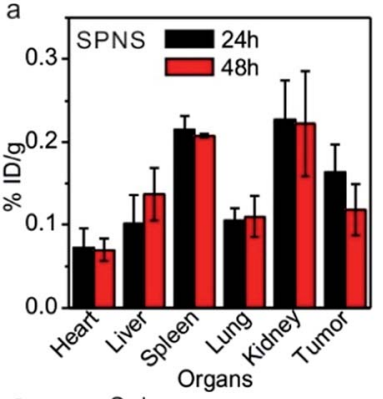

c

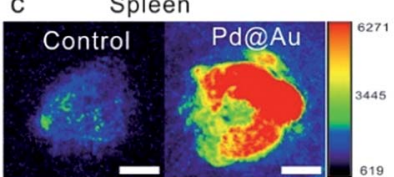

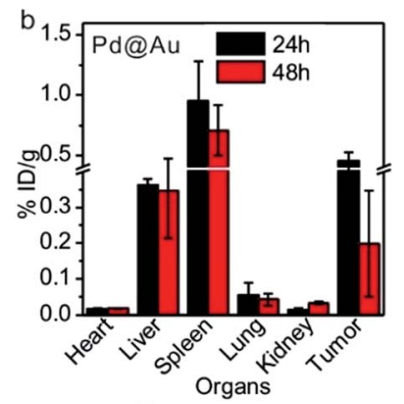

Tumor

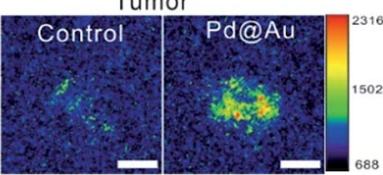

Fig. 5 Biodistribution of SPNS (a) and Pd@Au (b) in rabbit orthotopic liver tumor models $(n=4)$. (c) PA imaging comparison of the spleen and tumor of PdaAu-injected and PBS-injected rabbits. The scale bar for (c) and (d) is $1 \mathrm{~cm}$.

be incorporated to perform the PTT in the rat subcutaneous tumor model. To visually observe the uptake of nanomaterials by W256 rat tumor cells, SPNS and Pd@Au which were surface modified with the dye of FITC were co-cultured with the tumor cells for $6 \mathrm{~h}$ in 24-well plates. Observed using a Fluorescence Inversion Microscope System, we found that both SPNS and

Pd@Au could be effectively taken in by W256 cells (Fig. S11†). At the same time, the cell toxicity and PTT effect of SPNS and Pd@Au on W256 cells were also measured by MTT assay. The cell activity remained at a high level when co-cultured with SPNS or Pd@Au in the absence of 808 nm laser irradiation, while the cell activity decreased to less than $30 \%$ with laser irradiation $\left(0.5 \mathrm{~W} \mathrm{~cm}^{-2}\right)$ for $5 \mathrm{~min}$. The favourable therapeutic efficiency on the cellular level predicted that the solid tumor in vivo might be cured by PTT depending on the photothermal conversion capability of SPNS and Pd@Au.

Before in vivo treatment, we first investigated if SPNS and Pd@Au accumulated in the subcutaneous tumor sites of rats after intravenous administration could still generate a good rising temperature effect, which was the precondition for PTT. To perform this, $5 \mathrm{mg} \mathrm{kg}{ }^{-1}$ SPNS or Pd@Au were intravenously injected into the subcutaneous tumor-bearing rats. At $48 \mathrm{~h}$ post injection, the rats were exposed to $808 \mathrm{~nm}$ laser $\left(0.5 \mathrm{~W} \mathrm{~cm}^{-2}\right)$ irradiation for $5 \mathrm{~min}$, and the temperature changes of the tumor sites were recorded using a visual IR camera. As observed from Fig. 4c, in the groups treated with SPNS and Pd@Au for $5 \mathrm{~min}$, the temperature of the tumor sites could reach about $55{ }^{\circ} \mathrm{C}$ (Fig. 4c), whereas for the group treated with only laser irradiation for $5 \mathrm{~min}$, the tumor surface temperature could only reach 33.6 ${ }^{\circ} \mathrm{C}$. The results suggested that SPNS and Pd@Au accumulated in the subcutaneous tumors of rats could still effectively convert the absorbed light energy into heat for PTT treatment.
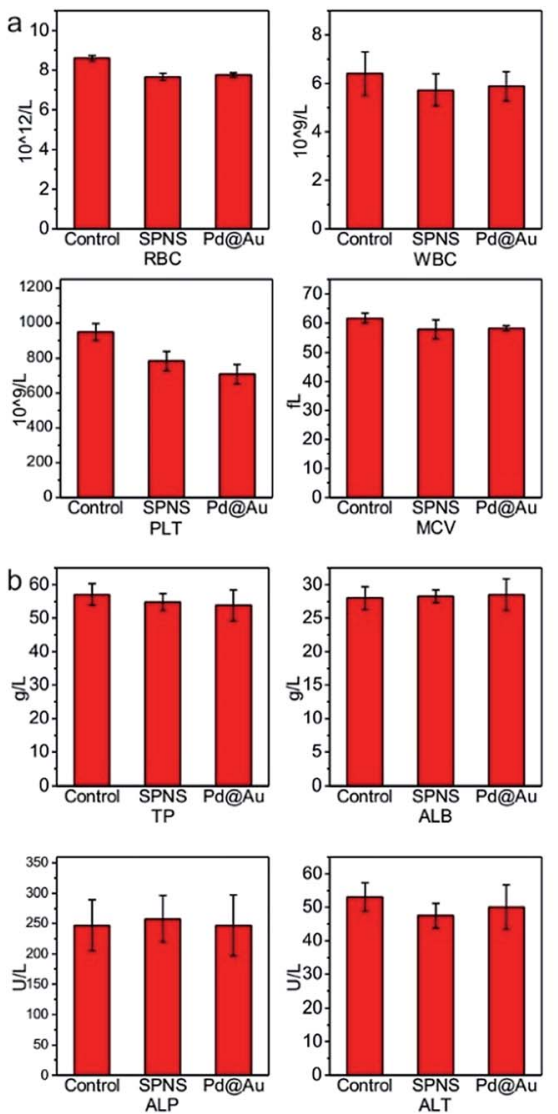
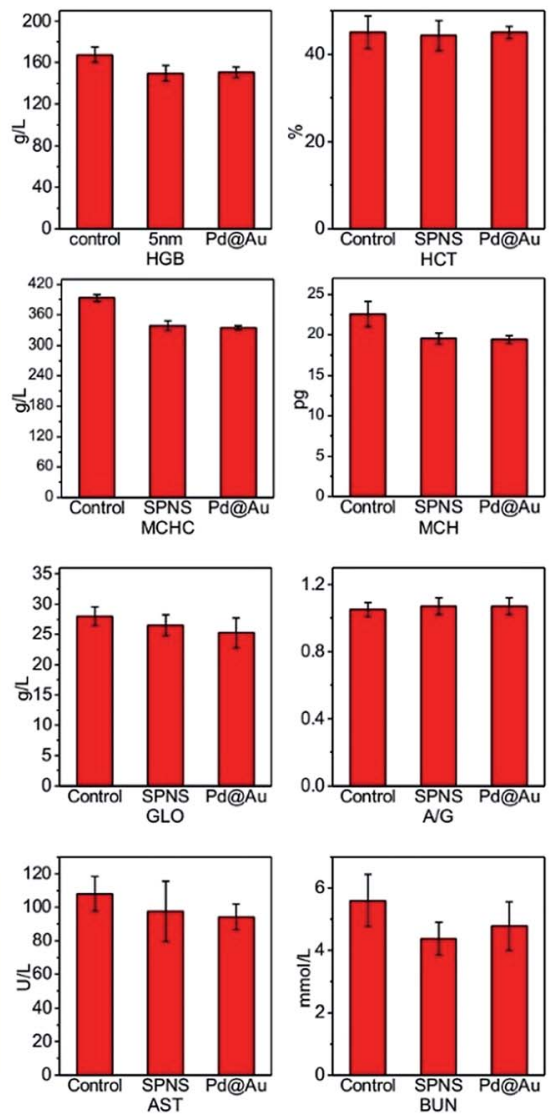

Fig. 6 The studies of biocompatibility: (a) blood routine and (b) blood serum biochemistry examination of SPNS and Pd@Au in rats. 
Encouraged by the good temperature rising effect in tumors caused by SPNS and Pd@Au with a laser, we further performed PTT in rats. Similarly, $48 \mathrm{~h}$ after injection with the same dose as above, the tumor sites were irradiated with an $808 \mathrm{~nm}$ laser $(0.5$ $\mathrm{W} \mathrm{cm}^{-2}$ ) for $5 \mathrm{~min}$. Meanwhile, the tumor-bearing rats in control or only-laser groups were intravenously injected with PBS and the latter were also treated with the same power density laser irradiation. Then the growth of tumors was photographed and tracked. As seen from Fig. $4 \mathrm{~d}$ and e, the tumors of rats in control or only-laser groups still kept growing during the observation whereas the tumors of rats which were treated with nanomaterials combined with laser irradiation gradually shrank and scabbed within 5 days and almost completely faded away at 10 days post-treatment. It could be concluded that combining the splendid photothermal conversion properties and passive tumor-targeting ability of 2D Pd-based nanomaterials, the effective killing of tumor cells by PTT at low power density could be realized. It was worth mentioning that the significant antitumor effect in subcutaneous tumor-bearing rats also proved that $2 \mathrm{D}$ Pd-based nanomaterials possessed great potential for clinical PTT application.

\section{In vivo biodistribution of SPNS and Pd@Au in the rabbit orthotopic liver tumor model}

Inspired by the passive accumulation effects with high efficiency of SPNS and Pd@Au in rat tumor models, we further attempted to investigate their accumulations in other bigger animal tumor models, such as rabbit orthotopic liver tumors which were made following a similar strategy to that of rat orthotopic liver tumors. SPNS and Pd@Au were intravenously administrated at the ear margin and then the rabbits were sacrificed at $24 \mathrm{~h}$ and $48 \mathrm{~h}$ post injection. The ICP-MS results indicated that SPNS could almost distribute in all investigated organs of rabbits, while Pd@Au mainly distributed in some RES organs such as the liver and spleen, possibly due to the bigger size of the nanomaterial and the higher enrichment in the RES organs (Fig. 5a and b). Particularly, both SPNS and Pd@Au demonstrated efficient accumulation in the rabbit orthotopic liver tumors, and the amounts at $24 \mathrm{~h}$ were $0.18 \%$ ID per $\mathrm{g}$ for SPNS and $0.46 \%$ ID per $g$ for Pd@Au, respectively. The accumulation of Pd@Au in rabbit orthotropic liver tumors was also confirmed by its photoacoustic (PA) imaging properties. From Fig. 5c, the PA signal of Pd@Au in tumors was obviously stronger than that in the control group which was injected with PBS, and the difference of signal intensity between the spleen and tumor was consistent with the ICP-MS results (Fig. 5b). The overall trends of biodistributions of SPNS and Pd@Au in rabbits were analogous to those in rats and mice. For SPNS, there was a certain distribution in each major organ especially in the kidney and tumor, while Pd@Au was much more concentrated in the spleen, liver and tumor. This situation was strongly related to the sizes of nanomaterials, which had been proved to
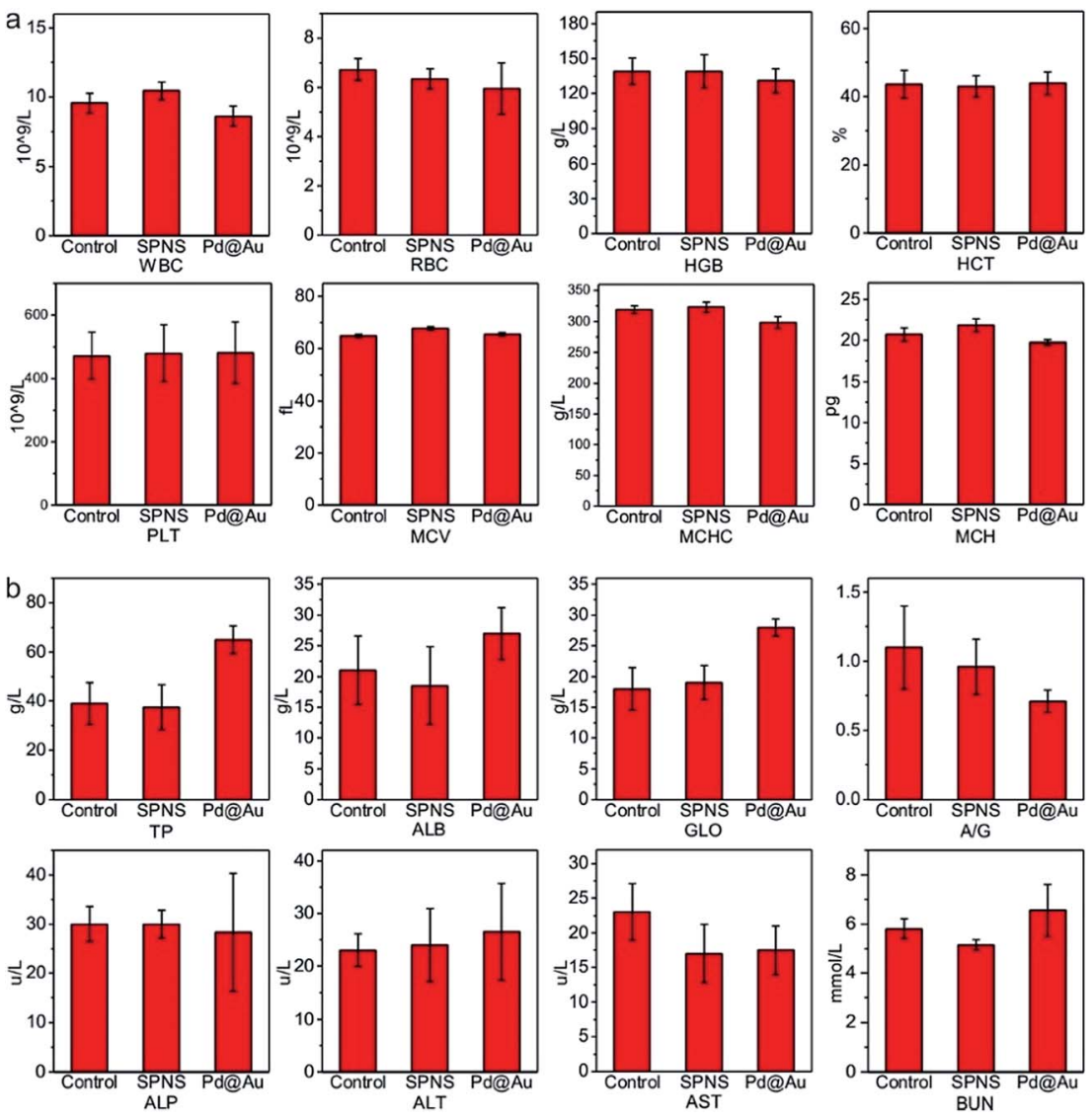

Fig. 7 The studies of biocompatibility: (a) blood routine and (b) blood serum biochemistry examination of SPNS and PdaAu in rabbits. 


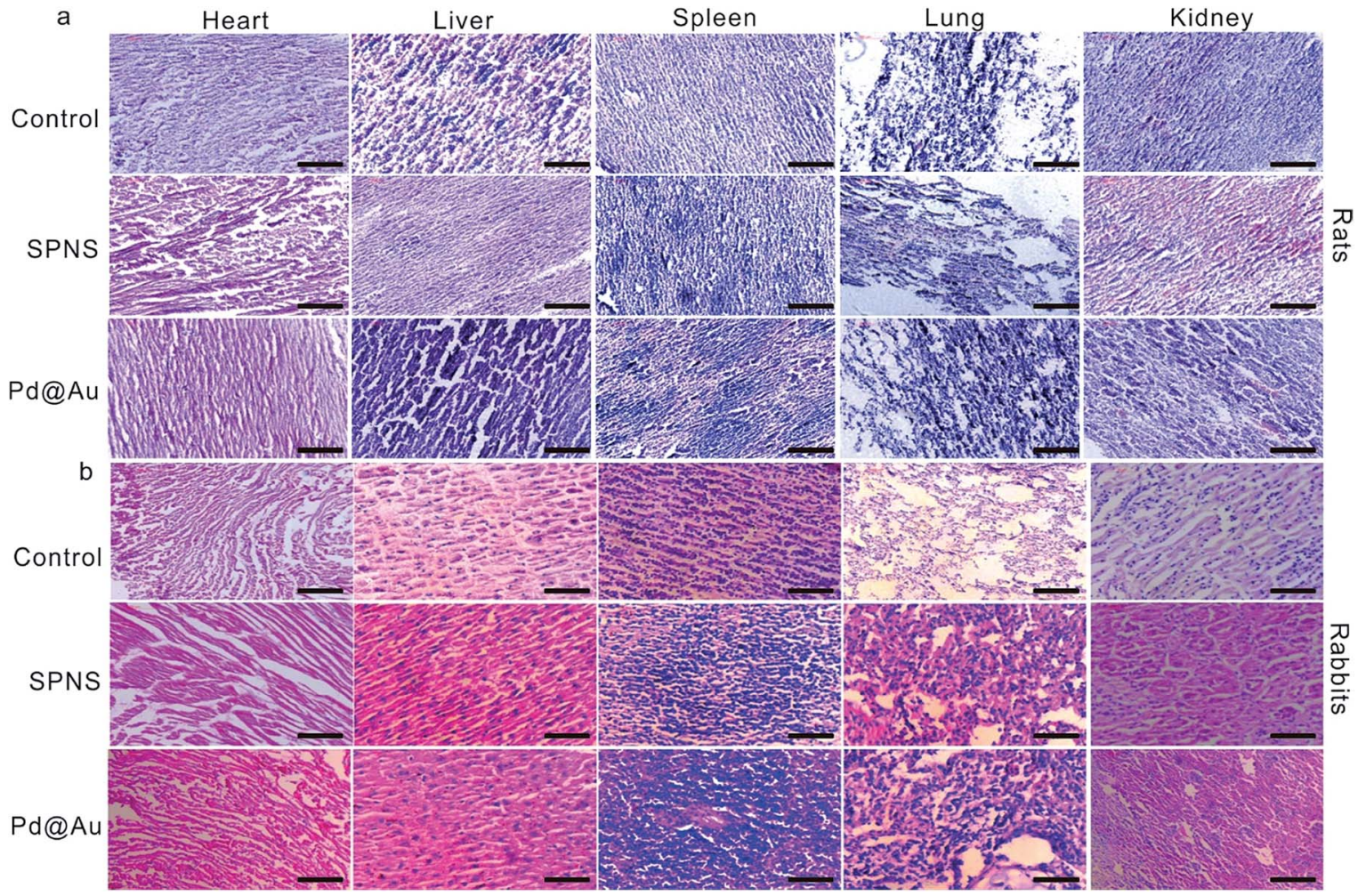

Fig. 8 Histological examination of SPNS and PdaAu in (a) rats and (b) rabbits, respectively. The scale bar is $50 \mu \mathrm{m}$.

have a great influence on the in vivo biobehaviors of $2 \mathrm{D}$ Pdbased nanomaterials in mice. In brief, these results suggested that the excellent EPR effect of 2D Pd-based nanomaterials was still retained in the rabbit orthotropic liver tumor model. Considering their capabilities for cellular internalization and in vitro killing VX2 cancer cells by PTT (Fig. S12 and S13†), 2Dbased nanomaterials hold promise for realizing PTT or PTTbased combined therapy in the rabbit tumor model, which further convincingly proved their value for clinical translation.

\section{Biosafety of SPNS and Pd@Au in rats and rabbits}

As mentioned above, SPNS and Pd@Au have excellent accumulation capacities in both rat and rabbit tumor sites by the EPR effect; however, it was still unclear if they could result in potential toxicity in some biochemical analysis including total proteins (TP), albumin (ALB), globulin (GLO), albumin/globulin (A/G), alkaline phosphatase (ALP), alanine aminotransferase (ALT), aspartate aminotransferase (AST) and blood urea nitrogen (BUN). From the results presented in Fig. 6 and 7, in the rat or rabbit models, all these detection indicators were changed within the normal range, which indicated that SPNS and Pd@Au would not cause an abnormal blood composition and side-effects on liver/kidney functions. What's more, histological analysis was performed to discuss whether SPNS and Pd@Au could give rise to inflammation or lesions on five major organs, including the heart, liver, spleen, lung and kidney. As shown in Fig. 8, the liver and spleen of rats and rabbits which were injected with the nanomaterial displayed a slight inflammation effect, possibly because of the high amount accumulation of nanomaterials in these two organs. Compared with the control group, other organs basically remained normal and there was no obvious damage. The above results manifested the superior biocompatibility of SPNS and Pd@Au for bigger animal models at the given dose, which was a valid supplement to the safety evaluation of 2D Pd-based nanomaterials.

\section{Conclusions}

Based on the prominent theranostic effects of 2D Pd-based nanomaterials on mouse tumor models before, in this work we successfully fabricated SD rat and New Zealand rabbit tumor models and systematically investigated the biobehaviors and biosafety of SPNS and $30 \mathrm{~nm}$ Pd@Au in these bigger animals. The results indicated that both SPNS and Pd@Au could efficiently accumulate in orthotopic liver tumors or subcutaneous tumors through the EPR effect. Utilizing the passive targeting ability of SPNS and Pd@Au in subcutaneous tumor together with their high photothermal conversion properties, highly efficient PTT was carried out to effectively eradicate tumors. Similar to the results obtained from mice, the urinary excretion and blood circulation of SPNS and Pd@Au were directly related to their sizes. Owing to the advantages of long blood circulation time and easy-elimination from the body, SPNS have been shown to have great clinical translation value. Moreover, SPNS and Pd@Au were proved to be safe in bigger animal models from the results of blood biochemical analysis and histopathology. 
Of course, compared with the results in mouse models, the EPR effect of nanomaterials was indeed affected by larger animal models, possibly because the larger animals have a more powerful RES and more complicated internal environments than mice. And the disadvantageous effect will be more obvious on larger-sized nanomaterials. In all, our work devoted to breaking the limits of animal models in previous experiments and pushing forward the clinical translation of 2D Pd-based nanomaterials may be a reference and guidance for other theranostic nanoplatforms.

\section{Experimental section}

\section{Construction of animal tumor models}

Female SD rats and New Zealand rabbits were obtained from Shanghai SLAC laboratory Animal Co. Ltd. All animal experiments were carried out in compliance with the guidelines for the Care and Use of Laboratory Animals of Xiamen University, and were approved by the Animal Ethics Committee of Xiamen University.

The Walker 256 (W256) cell line was kindly provided by Mengchao Hepatobiliary Hospital of Fujian Medical University. The orthotropic liver cancer tumor model of SD rats was generated by surgical operation. To be specific, the rats were anesthetized with chloral hydrate $(10 \%, \mathrm{w} / \mathrm{v})$ and then their abdominal area was shaved and scrubbed with ethanol (70\%, v/ v) for sterilization. An incision was made in the abdomen and 50 $\mu \mathrm{L}$ of the inoculation mixture that contained $4 \times 10^{6} \mathrm{~W} 256$ cells in culture medium was injected slowly into the left part of the liver. After finishing the injection, the abdominal wall was closed in layers and the rats revived in about $3 \mathrm{~h}$. After a week, the single tumor will grow to about $27 \mathrm{~mm}^{3}$. The subcutaneous tumor model of SD rats was made by subcutaneous injection of W256 cells $\left(\sim 4 \times 10^{6}\right.$ cells in $400 \mu \mathrm{L}$ phosphate buffer solution (PBS)) near the right leg of each rat. And the size of the tumor will reach around $200 \mathrm{~mm}^{3}$ after 6 days.

The procedure for fabricating the rabbit orthotropic liver tumor model was similar to that of rats. The tumor was predeveloped by subcutaneous injection of VX2 cells into the back of nude mice and then cut into small pieces. The rabbits were anesthetized and the tumor pieces were embedded into the liver. After 15-20 days, the rabbit orthotropic liver tumor will approximately reach $50 \mathrm{~mm}^{3}$.

\section{Synthesis and modification of SPNS and Pd@Au}

For the synthesis of SPNS, palladium acetylacetonate $(10 \mathrm{mg})$, polyvinyl pyrrolidone (PVP, $32 \mathrm{mg}$ ) and $\mathrm{NaBr}$ (10.6 mg) were mixed together with $N, N$-dimethylpropionamide $(2 \mathrm{~mL})$ and deionized (DI) water ( $4 \mathrm{~mL}$ ). After standing for $12 \mathrm{~h}$, the solution was transferred into a $60 \mathrm{~mL}$ glass pressure vessel. The vessel was charged with 1 bar $\mathrm{CO}$ and heated from room temperature to $100{ }^{\circ} \mathrm{C}$ within $30 \mathrm{~min}$ and kept at $100{ }^{\circ} \mathrm{C}$ for $2 \mathrm{~h}$. When the vessel was cooled to room temperature, dark blue products (SPNS) were collected and stored at $4{ }^{\circ} \mathrm{C}$ for further use.

30 nm Pd@Au nanoplates were synthesized by a seed growing method. Typically, about $30 \mathrm{~nm}$ Pd NSs were washed with acetone-ethanol and dispersed in DI water, then the solution was mixed with $\mathrm{AuPPh}_{3} \mathrm{Cl}\left(10 \mathrm{mg} \mathrm{mL}^{-1}\right.$ in $N, N$-dimethylformamide) to give a molar ratio of $\mathrm{Au}: \mathrm{Pd} \approx 10$. Hydrazine was chosen as the reductant and added dropwise into the above mixture under stirring. After standing at room temperature for $12 \mathrm{~h}$, the products were collected and stored at $4{ }^{\circ} \mathrm{C}$ for further use.

For the surface modification of mPEG-SH, both SPNS (1 mg) and Pd@Au NSs (1 mg) were washed with acetone-ethanol and dispersed in PBS, respectively. Then mPEG-SH (15 mg) was added and stirred for $2 \mathrm{~h}$. Free mPEG-SH was removed by ultrafiltration.

\section{Characterization of SPNS and Pd@Au}

The SPNS and Pd@Au NSs were dissolved in ethanol for Transmission electron microscopy (TEM) imaging by using a JEM-1400 TEM operating at $300 \mathrm{KV}$. The UV-Vis-NIR absorption spectra of the nanomaterials were obtained by using a Cary$5000 \mathrm{UV} / \mathrm{Vis} / \mathrm{NIR}$ spectrophotometer. The concentration of SPNS and Pd@Au aqueous solutions was 100 ppm, respectively.

\section{In vivo pharmacokinetics and biodistribution studies of SPNS- PEG and Pd@Au-PEG in rats}

Each rat (weight $\approx 180 \mathrm{~g}$ ) was injected with $0.5 \mathrm{~mL}$ PBS solution containing $1 \mathrm{mg}$ nanomaterial in the tail vein. For pharmacokinetic studies, 30-50 $\mu \mathrm{L}$ blood samples from the tail vein were collected at different time points post injection $(n=4$ for each group). For the tissue distribution study of the rat orthotropic liver tumor model, eight rats which were pre-injected with nanomaterials were divided into two groups and sacrificed at 24 $\mathrm{h}$ and $48 \mathrm{~h}$ post injection, respectively. The major organs including the heart, liver, spleen, lung, kidney and tumor were collected and cut into small parts as tissue samples. The blood and tissue samples were digested by using a mixture of $\mathrm{H}_{2} \mathrm{O}_{2}$ and $\mathrm{HNO}_{3}$. Inductively coupled plasma mass spectrometry (ICPMS) was used to detect Pd amounts in blood and tissue samples.

The tissue distribution assay of SPNS and Pd@Au in the rat subcutaneous tumor model followed the same protocol as the orthotropic liver tumor model except that the time point of detection was changed to $2 \mathrm{~h}, 12 \mathrm{~h}, 24 \mathrm{~h}$ and $48 \mathrm{~h}$, respectively ( $n$ $=4$ for each group).

The procedure of the biodistribution study in rabbits was also same as in the rat orthotropic liver tumor model. Each rabbit was approximately $3 \mathrm{~kg}$ in weight, tissue samples were collected at $24 \mathrm{~h}$ and $48 \mathrm{~h}$ post injection ( $n=4$ for each group).

\section{Urinary and faeces excretion of SPNS and Pd@Au from rats}

To investigate if the administrated nanomaterials could be efficiently excreted from rats through urine or faeces, the rats that were intravenously injected with SPNS or $30 \mathrm{~nm} \mathrm{Pd@Au}$ were solely kept in metabolism cages and their urine or faeces samples were collected at different time points for detecting the Pd amounts by ICP-MS. 4 rats were used in each experimental group. 


\section{In vivo fluorescence imaging of rats}

Cy5.5, which is widely used for live animal imaging, was chosen as the fluorescence indicator to label nanomaterials in our study. In brief, $15 \mathrm{mg}$ SH-PEG $(5 \mathrm{~K})-\mathrm{NH}_{2}$ was added to $1 \mathrm{~mL}$ SPNS or Pd@Au PBS solution which contains $1 \mathrm{mg}$ SPNS or Pd@Au, and after mixing for $6 \mathrm{~h}$, unloaded SH-PEG (5 K)- $\mathrm{NH}_{2}$ was removed by centrifugation. $0.5 \mathrm{mg}$ Cy5.5 was employed to modify SPNS or Pd@Au. The extra Cy5.5 was removed by dialysis via a dialysis bag (the CE membrane with a molecular weight cut-off of 2000). The final product was dispersed in PBS for tail-vein injection. The experimental rats were anesthetized at 2, 24 and $48 \mathrm{~h}$ post injection and the accumulation of SPNS and Pd@Au in tumors was observed by fluorescence imaging using a live animal optical imaging system (IVIS Lumina II).

\section{Cell uptake of nanomaterials and in vitro PTT}

Previous studies have indicated that 2D Pd nanomaterials could effectively be taken up by many kinds of cancer cells and play a therapeutic role. ${ }^{20}$ Could SPNS or Pd@Au be taken up by W256 cells (rat-derived cancer cell) or VX2 cells (rabbit-derived cancer cells) to achieve the PTT effect? To test this, W256 cells and VX2 cells were treated with dye (Lyso-Tracker Red, 4',6-diamidino-2phenylindole (DAPI) and fluorescein isothiocyanate (FITC))modified SPNS or Pd@Au for $6 \mathrm{~h}$. Then the cell uptake of nanomaterials was observed using a Fluorescence Inversion Microscope System (Eclipse Ti-U).

For in vitro photothermal therapy, the W256 cells were cultured in RPMI 1640 medium mixed with 10\% fetal bovine serum (FBS), penicillin (100 $\left.\mathrm{U} \mathrm{mL}^{-1}\right)$, and streptomycin $(100 \mu \mathrm{g}$ $\mathrm{mL}^{-1}$ ) at $37{ }^{\circ} \mathrm{C}$ in a humidified air atmosphere with $5 \% \mathrm{CO}_{2}$. The cells were seeded in two 96-well plates at $10^{5}$ cells/well. One plate was used for cytotoxicity assay without laser irradiation, and the other plate was under the same experimental conditions with laser irradiation at a power density of $0.5 \mathrm{~W} \mathrm{~cm}^{-2}$ for 5 min. Then MTT assay was performed to evaluate the cytotoxicity of the nanomaterial and in vitro PTT effect.

\section{In vivo PTT}

Female SD subcutaneous tumor-bearing rats were intravenously injected with SPNS or Pd@Au at a volume of $500 \mu \mathrm{L}(2 \mathrm{mg}$ $\left.\mathrm{mL}^{-1}\right)$. At $48 \mathrm{~h}$ post-injection, $\mathrm{SD}$ rats were anesthetized by intraperitoneal injection of $0.8 \mathrm{~mL}$ chloral hydrate $(40 \% \mathrm{w} / \mathrm{w})$ and the tumors were exposed to $0.5 \mathrm{~W} \mathrm{~cm}^{-2} 808 \mathrm{~nm}$ laser irradiation for $5 \mathrm{~min}$, the rats which were injected with PBS and also irradiated as before were assigned to the only-laser group. And the rats in the control group were all only treated with PBS. The growing states of tumors were recorded by taking photos and measuring with a vernier caliper.

\section{Blood serum analysis and histological examination}

The blood serum samples were collected from the janitrix when the rats or rabbits were sacrificed. The organs for histological examination were also derived from the step above. The blood serum analysis was finished in Mengchao Hepatobiliary Hospital of Fujian Medical University. For histological examination, the organs were dehydrated and cut into slices, and stained with hematoxylin \& eosin (H\&E). The final samples were observed and examined using a digital microscope.

\section{Statistical analysis}

All data were presented in this work as the mean \pm standard deviation (SD). The statistical analyses were all carried out by using Origin 2019 software. Asterisks stand for significant differences $\left({ }^{*} P<0.05, * * P<0.01\right.$, and $\left.{ }^{* * *} P<0.001\right)$.

\section{Conflicts of interest}

There are no conflicts to declare.

\section{Acknowledgements}

The work was financially supported by the National Basic Research Foundation (973) of China (2014CB932004), the National Key R\&D Program of China (2017YFA02073022), the National Natural Science Foundation of China (21731005, 21420102001, and 21721001), the Natural Science Foundation of Fujian Province (No. 2016J01073), and the State Key Laboratory for Chemistry and Molecular Engineering of Medicinal Resources (Guangxi Normal University, CMEMR 2018-B10).

\section{Notes and references}

1 B. Pelaz, C. Alexiou, R. A. Alvarez-Puebla, F. Alves, A. M. Andrews, S. Ashraf, L. P. Balogh, L. Ballerini, A. Bestetti, C. Brendel, S. Bosi, M. Carril, W. C. Chan, C. Chen, X. Chen, X. Chen, Z. Cheng, D. Cui, J. Du, C. Dullin, A. Escudero, N. Feliu, M. Gao, M. George, Y. Gogotsi, A. Grunweller, Z. Gu, N. J. Halas, N. Hampp, R. K. Hartmann, M. C. Hersam, P. Hunziker, J. Jian, X. Jiang, P. Jungebluth, P. Kadhiresan, K. Kataoka, A. Khademhosseini, J. Kopecek, N. A. Kotov, H. F. Krug, D. S. Lee, C. M. Lehr, K. W. Leong, X. J. Liang, M. Ling Lim, L. M. Liz-Marzan, X. Ma, P. Macchiarini, H. Meng, H. Mohwald, P. Mulvaney, A. E. Nel, S. Nie, P. Nordlander, T. Okano, J. Oliveira, T. H. Park, R. M. Penner, M. Prato, V. Puntes, V. M. Rotello, A. Samarakoon, R. E. Schaak, Y. Shen, S. Sjoqvist, A. G. Skirtach, M. G. Soliman, M. M. Stevens, H. W. Sung, B. Z. Tang, R. Tietze, B. N. Udugama, J. S. VanEpps, T. Weil, P. S. Weiss, I. Willner, Y. Wu, L. Yang, Z. Yue, Q. Zhang, Q. Zhang, X. E. Zhang, Y. Zhao, X. Zhou and W. J. Parak, ACS Nano, 2017, 11, 2313-2381.

2 B. Sumer and J. Gao, Nanomedicine, 2008, 3, 137-140.

3 T. Lammers, S. Aime, W. E. Hennink, G. Storm and F. Kiessling, Acc. Chem. Res., 2011, 44, 1029-1038.

4 N. Ahmed, H. Fessi and A. Elaissari, Drug Discovery Today, 2012, 17, 928-934.

5 X. Sun, W. Cai and X. Chen, Acc. Chem. Res., 2015, 48, 286294.

6 M. Liong, J. Lu, M. Kovochich, T. Xia, S. G. Ruehm, A. E. Nel, F. Tamanoi and J. I. Zink, ACS Nano, 2008, 2, 889-896. 
7 K. Yang, L. Feng, X. Shi and Z. Liu, Chem. Soc. Rev., 2013, 42, 530-547.

8 L. Z. He, Y. Y. Huang, H. L. Zhu, G. H. Pang, W. J. Zheng, Y. S. Wong and T. F. Chen, Adv. Funct. Mater., 2014, 24, 2754-2763.

9 X. Yang, M. Yang, B. Pang, M. Vara and Y. Xia, Chem. Rev., 2015, 115, 10410-10488.

10 Z. Zhang, C. Liu, J. Bai, C. Wu, Y. Xiao, Y. Li, J. Zheng, R. Yang and W. Tan, ACS Appl. Mater. Interfaces, 2015, 7, 6211-6219.

11 T. Shanmugasundaram, M. Radhakrishnan, V. Gopikrishnan, K. Kadirvelu and R. Balagurunathan, Nanoscale, 2017, 9, 16773-16790.

12 Z. Liu, S. Zhang, H. Lin, M. Zhao, H. Yao, L. Zhang, W. Peng and Y. Chen, Biomaterials, 2018, 155, 54-63.

13 R. Weissleder, Nat. Rev. Cancer, 2002, 2, 11-18.

14 X. Chen, J. Li, Y. Huang, J. Wei, D. Sun and N. Zheng, Biomater. Sci., 2017, 5, 2448-2455.

15 Z. Li, Y. L. Song, Y. H. Yang, L. Yang, X. H. Huang, J. H. Han and S. F. Han, Chem. Sci., 2012, 3, 2941-2948.

16 D. A. Jasim, C. Menard-Moyon, D. Begin, A. Bianco and K. Kostarelos, Chem. Sci., 2015, 6, 3952-3964.

17 B. Pili, L. H. Reddy, C. Bourgaux, S. Lepetre-Mouelhi, D. Desmaele and P. Couvreur, Nanoscale, 2010, 2, 1521-1526.

18 M. Chen, Z. Guo, Q. Chen, J. Wei, J. Li, C. Shi, D. Xu, D. Zhou, X. Zhang and N. Zheng, Chem. Sci., 2018, 9, 4268-4274.

19 C. Liang, L. Xu, G. Song and Z. Liu, Chem. Soc. Rev., 2016, 45, 6250-6269.

20 X. L. Chen, S. G. Shi, J. P. Wei, M. Chen and N. F. Zheng, Sci. Bull., 2017, 62, 579-588.

21 L. Cheng, J. Liu, X. Gu, H. Gong, X. Shi, T. Liu, C. Wang, X. Wang, G. Liu, H. Xing, W. Bu, B. Sun and Z. Liu, Adv. Mater., 2014, 26, 1886-1893.
22 G. K. Das, D. S. Anderson, C. D. Wallis, S. A. Carratt, I. M. Kennedy and L. S. Van Winkle, Nanoscale, 2016, 8, 11518-11530.

23 H. J. Paek, Y. J. Lee, H. E. Chung, N. H. Yoo, J. A. Lee, M. K. Kim, J. K. Lee, J. Jeong and S. J. Choi, Nanoscale, 2013, 5, 11416-11427.

24 T. W. Prow, Wiley Interdiscip. Rev.: Nanomed. Nanobiotechnol., 2010, 2, 317-333.

25 G. Terentyuk, E. Panfilova, V. Khanadeev, D. Chumakov, E. Genina, A. Bashkatov, V. Tuchin, A. Bucharskaya, G. Maslyakova, N. Khlebtsov and B. Khlebtsov, Nano Res., 2014, 7, 325-337.

26 L. Tan, S. Wang, K. Xu, T. Liu, P. Liang, M. Niu, C. Fu, H. Shao, J. Yu, T. Ma, X. Ren, H. Li, J. Dou, J. Ren and X. Meng, Small, 2016, 12, 2046-2055.

27 Y. Z. Huang, X. L. Chen, S. G. Shi, M. Chen, S. H. Tang, S. G. Mo, J. P. Wei and N. F. Zheng, Sci. China: Chem., 2015, 58, 1753-1758.

28 M. Chen, S. Chen, C. He, S. Mo, X. Wang, G. Liu and N. Zheng, Nano Res., 2016, 10, 1234-1248.

29 S. Shi, X. Chen, J. Wei, Y. Huang, J. Weng and N. Zheng, Nanoscale, 2016, 8, 5706-5713.

30 S. Tang, M. Chen and N. Zheng, Small, 2014, 10, 3139-3144.

31 M. Chen, S. Tang, Z. Guo, X. Wang, S. Mo, X. Huang, G. Liu and N. Zheng, Adv. Mater., 2014, 26, 8210-8216.

32 S. Shi, Y. Huang, X. Chen, J. Weng and N. Zheng, ACS Appl. Mater. Interfaces, 2015, 7, 14369-14375.

33 M. Longmire, P. L. Choyke and H. Kobayashi, Nanomedicine, 2008, 3, 703-717.

34 M. Yu and J. Zheng, ACS Nano, 2015, 9, 6655-6674. 